\title{
Systematic Analysis of Endometrial Cancer-Associated Hub Proteins Based on Text Mining
}

\author{
Huiqiao Gao and Zhenyu Zhang \\ Department of Obstetrics and Gynecology, Beijing Chao-yang Hospital, Capital Medical University, Beijing 100020, China \\ Correspondence should be addressed to Zhenyu Zhang; zhenyuzhang2010@163.com
}

Received 2 July 2015; Accepted 11 August 2015

Academic Editor: Mona A. El-Bahrawy

Copyright (c) 2015 H. Gao and Z. Zhang. This is an open access article distributed under the Creative Commons Attribution License, which permits unrestricted use, distribution, and reproduction in any medium, provided the original work is properly cited.

\begin{abstract}
Objective. The aim of this study was to systematically characterize the expression of endometrial cancer- (EC-) associated genes and to analysis the functions, pathways, and networks of EC-associated hub proteins. Methods. Gene data for EC were extracted from the PubMed (MEDLINE) database using text mining based on NLP. PPI networks and pathways were integrated and obtained from the KEGG and other databases. Proteins that interacted with at least 10 other proteins were identified as the hub proteins of the EC-related genes network. Results. A total of 489 genes were identified as EC-related with $P<0.05$, and 32 pathways were identified as significant $(P<0.05$, FDR $<0.05)$. A network of EC-related proteins that included 271 interactions was constructed. The 17 proteins that interact with 10 or more other proteins $(P<0.05$, FDR $<0.05)$ were identified as the hub proteins of this PPI network of EC-related genes. These 17 proteins are EGFR, MET, PDGFRB, CCND1, JUN, FGFR2, MYC, PIK3CA, PIK3R1, PIK3R2, KRAS, MAPK3, CTNNB1, RELA, JAK2, AKT1, and AKT2. Conclusion. Our data may help to reveal the molecular mechanisms of EC development and provide implications for targeted therapy for EC. However, corrections between certain proteins and EC continue to require additional exploration.
\end{abstract}

\section{Introduction}

Endometrial cancer is one of the most common gynecologic malignancies, and the incidence of this cancer continues to increase [1]. During the prior several decades, progress in molecular biology has improved our understanding of the occurrence and development of EC. It has been established that the biological behavior of tumors is controlled by functional proteins within cells and the signaling pathways in which these proteins are involved. Therefore, studies on the structure and function of hub proteins in signaling pathways may be valuable for diagnosing EC and for determining targeted therapies for this disease. To date, research has examined a large number of EC-related genes and proteins that could potentially be used as biomarkers or targets for diagnosis or treatment $[2,3]$. However, most published papers regarding EC have focused on only a handful of genes and proteins. Although the research objectives of molecular biology are shifting from single genes or proteins to genomics or proteomics, there are a limited number of systematic studies of whole-genome expression in the context of EC.
At present, text mining (TM) technology is widely used in biomedical research to extract information from large quantities of biomedical literature and construct databases of disease-related genes, proteins, and molecular interactions $[4,5]$. In this study, we systematically characterized the expression of EC-associated genes by mining data from the PubMed document retrieval system. In addition, we used bioinformatics methods to analyze the functions, pathways, and networks of relevant hub proteins.

\section{Materials and Methods}

The extraction of data by TM was based on natural language processing (NLP). Using "Endometrial Cancer" and "Endometrium Carcinoma" as search terms, we searched the PubMed database for article abstracts published before March 2014 and formatted the documents that were obtained. Genes and proteins that appeared in the abstracts of these documents were located and tagged using ABNER (A Biomedical Named Entity Recognizer; an open source tool for automatically tagging genes, proteins, and other entity 
TABLE 1: The 20 most significant EC-related genes based on text mining.

\begin{tabular}{|c|c|c|c|}
\hline Gene & Description & Count & $P$ value \\
\hline PGR & Progesterone receptor & 323 & 0 \\
\hline TP53 & Tumor protein p53 & 296 & 0 \\
\hline MLH1 & mutL homolog 1 & 150 & 0 \\
\hline PTEN & Phosphatase and tensin homolog & 130 & 0 \\
\hline MSH2 & mutS homolog 2 & 112 & 0 \\
\hline VEGFA & Vascular endothelial growth factor A & 82 & 0 \\
\hline ERBB2 & erb-b2 receptor tyrosine kinase 2 (HER2) & 77 & 0 \\
\hline MSH6 & mutS homolog 6 & 75 & 0 \\
\hline EGFR & Epidermal growth factor receptor & 68 & 0 \\
\hline MKI67 & Antigen identified by monoclonal antibody Ki-67 & 66 & $4.80 E-09$ \\
\hline BCL2 & B-cell CLL/lymphoma 2 & 54 & 0 \\
\hline CCND1 & Cyclin D1 & 53 & $1.02 E-08$ \\
\hline ESR1 & Estrogen receptor 1 & 48 & 0 \\
\hline TCEAL1 & Transcription elongation factor A (SII)-like 1 & 47 & 0 \\
\hline CDKN2A & Cyclin-dependent kinase inhibitor 2A (p16) & 39 & 0 \\
\hline CYP19A1 & Cytochrome P450, family 19 , subfamily A, polypeptide 1 & 39 & 0 \\
\hline INS & insulin & 36 & 0 \\
\hline PTGS2 & Prostaglandin-endoperoxide synthase 2 (COX2) & 34 & 0 \\
\hline PMS2 & Postmeiotic segregation increased 2 & 33 & 0 \\
\hline PCNA & Proliferating cell nuclear antigen & 32 & 0 \\
\hline
\end{tabular}

names in text) [6]. Gene names were normalized based on the Entrez Gene database (the National Center for Biotechnology Information's database for gene-specific information) [7]. The frequency at which each gene occurred was then counted. A hypergeometric distribution was used to calculate the probabilities that genes would be cocited with EC at frequencies higher than theoretical expectations; genes of which $P<0.05$ were considered relevant.

Gene ontology (GO) analysis was performed using GSEABase software package from the $\mathrm{R}$ statistical platform (http://www.r-project.org/). Genes were classified by biological process, cellular component, and molecular function. The EC-related protein-protein interaction (PPI) network was integrated from the KEGG (Kyoto Encyclopedia of Genes and Genomes), MIPS (Munich Information Center for Protein Sequences), and PubMed databases. GenMAPP v2.1 was used to map EC-related genes to the KEGG database to determine the pathways in which these genes were involved. A threshold of 0.05 was established for $P$ values and false discovery rate (FDR).

\section{Results}

3.1. EC-Related Genes and GO Analysis. After the retrieval of documents from PubMed, 15157 abstracts were examined, and 832 genes were obtained. Eventually, a total of 489 genes were identified as EC-related with $P<0.05$; among these genes, PGR, TP53, and MLH1 were mentioned most frequently. Table 1 lists the 20 most significant EC-related genes.

Classification results for biological processes, cellular components, and molecular functions by GO analysis are presented in Table 2. Developmental processes, protein metabolism, and signal transduction were the major biological processes associated with EC-related genes; with respect to molecular function, the primary activities of these genes included signal transduction, nucleic acid binding, and transcriptional regulation. These genes were related to various cellular components, including the nucleus, plasma membrane, and nonstructural extracellular matrix.

3.2. Pathway and PPI Analysis. Following pathway analysis, 32 pathways were identified as significant $(P<0.05$, FDR $<$ $0.05)$; among these pathways, the cytokine-cytokine receptor interaction, MAPK, and focal adhesion signaling pathways involved the largest number of genes. Table 3 lists the 20 most significant EC-related pathways.

We constructed a network of EC-related proteins that included 271 interactions (Figure 1). The 17 proteins that interact with at least 10 other proteins $(P<0.05$, FDR $<0.05)$ were identified as the hub proteins of the EC-related PPI network. These proteins are EGFR, MET, PDGFRB, CCND1, JUN, FGFR2, MYC, PIK3CA, PIK3R1, PIK3R2, KRAS, MAPK3, CTNNB1, RELA, JAK2, AKT1, and AKT2 (Figure 2). EGFR, which interacts with 33 other proteins, was the EC-related protein that exhibited the greatest number of interactions.

\section{Discussion}

In the present study, by extracting information from biomedical literature, we obtained a dataset of EC-related proteins and identified 17 hub proteins. Most relationships between EC and certain hub proteins, such as EGFR, IGF1R, and MET, 
TABLE 2: Classification results for biological processes, cellular components, and molecular functions by GO analysis.

\begin{tabular}{|c|c|c|}
\hline Term & Count & $P$ value \\
\hline \multicolumn{3}{|l|}{ Biological process } \\
\hline Cell cycle and proliferation & 224 & $4.05 E-11$ \\
\hline Stress response & 160 & $5.51 E-11$ \\
\hline Developmental processes & 336 & $8.54 E-11$ \\
\hline RNA metabolism & 188 & 0.00031 \\
\hline DNA metabolism & 67 & 0 \\
\hline Protein metabolism & 254 & $1.07 E-10$ \\
\hline Other metabolic processes & 229 & $2.58 E-10$ \\
\hline Cell organization and biogenesis & 178 & $7.72 E-11$ \\
\hline Cell-cell signaling & 44 & $8.21 E-08$ \\
\hline Signal transduction & 245 & 0.00089 \\
\hline Cell adhesion & 51 & 0.00284 \\
\hline Death & 141 & $2.77 E-11$ \\
\hline Other biological processes & 436 & $5.94 E-06$ \\
\hline \multicolumn{3}{|l|}{ Molecular function } \\
\hline Transcription regulatory activity & 107 & $8.46 E-10$ \\
\hline Signal transduction activity & 240 & $3.60 E-05$ \\
\hline Enzyme regulator activity & 48 & 0.01638 \\
\hline Nucleic acid binding activity & 194 & $2.53 E-07$ \\
\hline Kinase activity & 84 & $1.32 E-08$ \\
\hline Other molecular function & 744 & $2.86 E-07$ \\
\hline \multicolumn{3}{|l|}{ Cellular component } \\
\hline Extracellular matrix & 34 & $1.42 E-05$ \\
\hline Nonstructural extracellular & 180 & $1.09 E-10$ \\
\hline Cytosol & 53 & $5.50 E-11$ \\
\hline Nucleus & 306 & $1.77 E-09$ \\
\hline Plasma membrane & 186 & 0.00014 \\
\hline Translational apparatus & 22 & 0.00148 \\
\hline Other cellular component & 446 & $9.02 E-08$ \\
\hline
\end{tabular}

have been extensively studied, and all of the aforementioned proteins are known to be closely related to the occurrence and development of EC. However, relative to these proteins, PDGFRB, FGFR2, MAPK3, and JAK2 have been reported less frequently in the context of EC.

4.1. PI3K and AKT. PI3K is a heterodimeric enzyme that consists of a regulatory subunit (p85) encoded by PIK3R1, PIK3R2, and PIK3R3 and a catalytic subunit (p110) encoded by PIK3CA, PIK3CB, and PIK3CD [8]. Mutations in PIK3CA, PIK3R1, and PIK3R2 occur at high rates in EC $[9,10]$. AKT is the downstream target gene of PI3K, and AKT1 and AKT2 are two subtypes of AKT. Based on data mining, we found that PI3K and AKT are involved in many pathways, including the focal adhesion pathway, the toll-like receptor signaling pathway, and, most notably, the PI3K/AKT signaling pathway. PI3K phosphorylates PIP2 to PIP3, which can activate AKT. Subsequently, activated AKT stimulates the regulation of cellular metabolism, growth and survival by CCND1, Myc, $\mathrm{NF}-\kappa \mathrm{B}$, and a variety of downstream factors [11]. AKT plays a key role in this pathway. The PI3K/AKT signaling pathway
TABLE 3: The 20 most significant pathways in which EC-related genes were involved.

\begin{tabular}{lcc}
\hline Term & Count & $P$ value \\
\hline Cytokine-cytokine receptor interaction & 64 & $1.95 E-09$ \\
MAPK signaling pathway & 62 & $2.91 E-08$ \\
Focal adhesion & 52 & $1.12 E-08$ \\
Cell cycle & 48 & $8.71 E-15$ \\
Regulation of actin cytoskeleton & 46 & $2.43 E-05$ \\
Jak-STAT signaling pathway & 39 & $2.25 E-06$ \\
Toll-like receptor signaling pathway & 36 & $3.72 E-10$ \\
Chemokine signaling pathway & 36 & 0.00170 \\
p53 signaling pathway & 34 & $1.63 E-14$ \\
Apoptosis & 33 & $3.75 E-10$ \\
T cell receptor signaling pathway & 33 & $1.55 E-07$ \\
Insulin signaling pathway & 33 & $3.00 E-05$ \\
ErbB signaling pathway & 32 & $1.77 E-09$ \\
Wnt signaling pathway & 32 & $6.43 E-04$ \\
Neurotrophin signaling pathway & 31 & $3.51 E-05$ \\
Natural killer cell-mediated cytotoxicity & 28 & 0.00168 \\
Steroid hormone biosynthesis & 26 & $9.99 E-13$ \\
Adherens junction & 24 & $7.63 E-06$ \\
Fc epsilon RI signaling pathway & 24 & $9.69 E-06$ \\
NOD-like receptor signaling pathway & 23 & $4.62 E-07$ \\
\hline
\end{tabular}

can inhibit cell apoptosis and promote cell proliferation [12]. In EC, molecular alterations lead to increased PI3K/AKT signaling; in particular, the dominant activation event is the loss of the PTEN protein, which is a tumor suppressor that negatively affects the PI3K signaling pathway [11, 13]. Many recent studies have demonstrated that the PI3K/AKT pathway is activated in all types of $\mathrm{EC}$ and that this activation is associated with the aggressiveness of this disease [14, 15]. Recently, certain PI3K/AKT pathway inhibitors have been evaluated in preclinical or early clinical trials [16].

4.2. RAS and MAPK. RAS is an oncogene that serves as a central focus for many signal transduction pathways associated with a high percentage of human tumors. Activating mutations in KRAS can be observed in EC [17]. A recent analysis of EC signal transduction indicated that KRAS mutation is associated with elevated phosphorylation of MEK1/2, ERK1/2, and p38MAPK [9]. In fact, many studies have indicated that the RAS/MAPK pathway is frequently upregulated in EC $[18,19]$. Moreover, KRAS also interacts with the PI3K pathway. Notably, KRAS-induced carcinogenesis can be inhibited when the interaction between RAS and the PI3K catalytic subunit P110 $\alpha$ is blocked in vitro [20]. In this study, we found that KRAS and MAPK were involved in many signaling pathways, such as the MAPK signaling pathway, pathways involved in regulating the actin cytoskeleton, and the ErbB signaling pathway. As a hub of various pathways, MAPK regulates a cascade of downstream genes that participate in cell proliferation and differentiation, including $\mathrm{Bcl}-2$, $\mathrm{c}-\mathrm{Myc}$, rock, and RSK2, among others. 


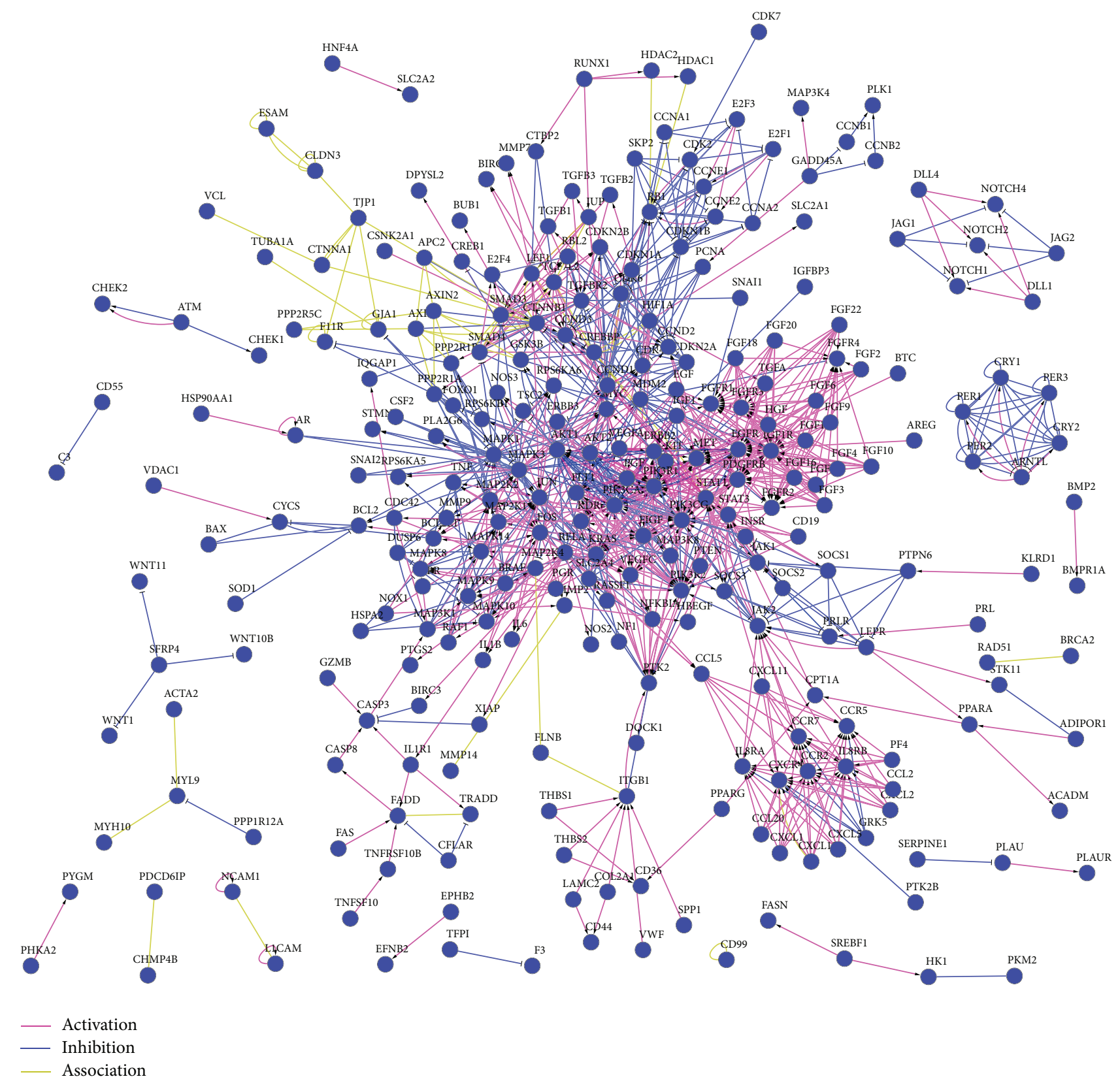

FIGURE 1: Network analysis of EC-related genes.

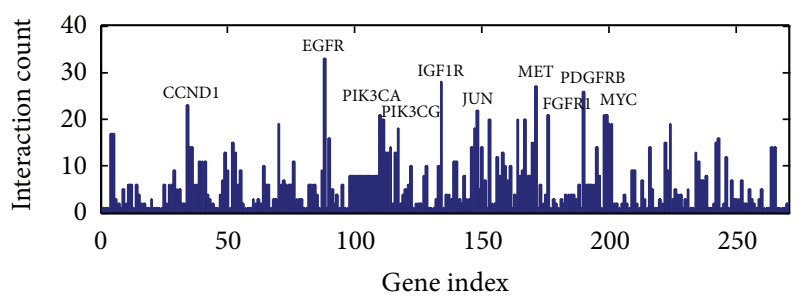

FIGURE 2: Hub proteins for EC.

4.3. FGFR2. FGFR2 is one type of fibroblast growth factor receptor and a member of the RTK family. RTKs are well known for their role in tumorigenesis [21]. In addition, it has been demonstrated that activating mutations in FGFR2 are associated with multiple types of tumors, including EC. By utilizing immunohistochemistry and PCR to examine FGFR2 expression and the presence of FGFR2 mutations in endometrial carcinoma, Gatius et al. determined that FGFR2 acted as an oncogene in EC and that FGFR2 expression was positively correlated with tumor stage and grade [22]. In our study, FGFR2 was mainly found to be involved in the MAPK signaling pathway and the regulation of the actin cytoskeleton. In fact, FGF signaling can activate several downstream pathways, including both the RAS-MAPK pathway and the PI3K-AKT pathway [23]. There has long 
been interest in FGFR inhibitors, and many studies have demonstrated that FGFR inhibition can block the progression of FGFR2-mutated EC [24, 25]. The targeting of FGFR2 is a possible treatment strategy for endometrial carcinoma.

4.4. PDGFRB. PDGF is a major mitogen that mediates the growth of fibroblasts, smooth muscle cells, and other cell. This protein also has significant effects on the angiogenesis of endothelial cells. PDGF exerts its biological effects by binding to its two receptors, $\alpha$-receptor (PDGFRA) and $\beta$ receptor (PDGFRB), which are located on the cell membrane. These PDGF receptors are also members of the RTK family. In vivo and in vitro research have indicated that the excessive expression of PDGF and PDGFR can be detected in breast, pancreas, colorectal, and other tumors [26, 27]. Liegl et al. demonstrated that PDFGRB can be detected in the endothelial cells of endometrial stromal sarcomas [28]. PDGFR-mediated signaling contributes to tumor angiogenesis, and PDGF can upregulate the expression of VEGF, which also has angiogenic effects. Our TM indicated that PDGFRB participated as an upstream factor in cytokinecytokine receptor interaction, the MAPK signaling pathway, focal adhesion, and the regulation of actin cytoskeleton. Moreover, the targeting of PDGFR to inhibit tumor cell signal transduction may play a crucial antitumor role $[29,30]$.

4.5. JAK2. JAK2, a member of the JAK family, is widely distributed in the cytoplasm. This protein is involved in signal transduction during hematopoiesis and in the immune system; in particular, JAK2 plays important roles in the production of red blood cells and the activation of immune cells. Research has demonstrated that JAK2 is associated with multiple tumors. The constitutive activation of JAK2 has been detected in many malignant solid tumors, such as colon cancer, head and neck cancer, leukemia, multiple myeloma, and other blood diseases [31-33]. Several JAK2 inhibitors are currently being evaluated in clinical trials in patients [34, 35]. JAK2 forms several signal transduction pathways in combination with multiple members of the STAT family; among these pathways, the JAK2-STAT3 pathway is particularly prominent. The JAK2-STAT3 signaling pathway, which mediates cell proliferation, differentiation, and apoptosis, is a focal point of the cellular signaling network and is closely associated with tumorigenesis [36]. However, there exists little research addressing the correlation between EC and JAK2-STAT3. The research of Liu et al. and Gao et al. indicated that the leptin can promote EC growth via activating the JAK2-STAT3 signal pathway in obese patient $[37,38]$. In our study, JAK2 not only participates in the JAKSTAT pathway but also can activate the downstream PI3KAKT pathway.

In summary, in this investigation, we systematically analyzed EC-related genes and identified certain hub proteins and their pathways and networks. This systematic study may help to reveal the molecular mechanisms of EC development. However, the study results were obtained based on TM, which only considered previously published literatures; thus, the correlations between certain proteins and EC require additional explorations. Moreover, our data also provide implications for targeted therapy for EC. After obtaining deeper insight into the EC-related signaling network, additional hub protein inhibitors with stronger specificities will be developed. Anyhow, multiple hub proteins-targeted drugs will have broad potential for tumor treatment.

\section{Conflict of Interests}

The authors declare that there is no conflict of interests regarding the publication of this paper.

\section{Acknowledgments}

This study was supported in part by the Chinese High-tech R\&D (863) Program. The authors also wish to express their gratitude to Shanghai Sensichip Co., Ltd., for bioinformatics analysis.

\section{References}

[1] R. Siegel, J. Ma, Z. Zou, and A. Jemal, "Cancer statistics, 2014," CA Cancer Journal for Clinicians, vol. 64, no. 1, pp. 9-29, 2014.

[2] S. S. Mihalj, I. Kuzmić-Prusac, S. Zekić-Tomaš, I. Šamija-Projić, and V. Čapkun, "Lipocalin-2 and matrix metalloproteinase-9 expression in high-grade endometrial cancer and their prognostic value," Histopathology, vol. 67, no. 2, pp. 206-215, 2015.

[3] J. Wang, A. Taylor, R. Showeil et al., "Expression profiling and significance of VEGF-A, VEGFR2, VEGFR3 and related proteins in endometrial carcinoma," Cytokine, vol. 68, no. 2, pp. 94-100, 2014.

[4] F. Zhu, P. Patumcharoenpol, C. Zhang et al., "Biomedical text mining and its applications in cancer research," Journal of Biomedical Informatics, vol. 46, no. 2, pp. 200-211, 2013.

[5] I. Spasić, J. Livsey, J. A. Keane, and G. Nenadić, “Text mining of cancer-related information: review of current status and future directions," International Journal of Medical Informatics, vol. 83, no. 9, pp. 605-623, 2014.

[6] B. Settles, "ABNER: an open source tool for automatically tagging genes, proteins and other entity names in text," Bioinformatics, vol. 21, no. 14, pp. 3191-3192, 2005.

[7] D. Maglott, J. Ostell, K. D. Pruitt, and T. Tatusova, "Entrez gene: gene-centered information at NCBI," Nucleic Acids Research, vol. 39, no. 1, pp. D52-D57, 2011.

[8] P. K. Vogt, J. R. Hart, M. Gymnopoulos et al., "Phosphatidylinositol 3-kinase: the oncoprotein," Current Topics in Microbiology and Immunology, vol. 347, no. 1, pp. 79-104, 2010.

[9] L. W. Cheung, B. T. Hennessy, J. Li et al., "High frequency of PIK3R1 and PIK3R2 mutations in endometrial cancer elucidates a novel mechanism for regulation of PTEN protein stability," Cancer Discovery, vol. 1, no. 2, pp. 170-185, 2011.

[10] M. L. Rudd, J. C. Price, S. Fogoros et al., "A unique spectrum of somatic PIK3CA (p110alpha) mutations within primary endometrial carcinomas," Clinical Cancer Research, vol. 17, no. 6, pp. 1331-1340, 2011.

[11] B. M. Slomovitz and R. L. Coleman, "The PI3K/AKT/mTOR pathway as a therapeutic target in endometrial cancer," Clinical Cancer Research, vol. 18, no. 21, pp. 5856-5864, 2012.

[12] M. Cully, H. You, A. J. Levine, and T. W. Mak, "Beyond PTEN mutations: the PI3K pathway as an integrator of multiple inputs 
during tumorigenesis," Nature Reviews Cancer, vol. 6, no. 3, pp. 184-192, 2006.

[13] B. Djordjevic, B. T. Hennessy, J. Li et al., "Clinical assessment of PTEN loss in endometrial carcinoma: immunohistochemistry outperforms gene sequencing," Modern Pathology, vol. 25, no. 5, pp. 699-708, 2012.

[14] R. A. Nout, T. Bosse, C. L. Creutzberg et al., "Improved risk assessment of endometrial cancer by combined analysis of MSI, PI3K-AKT, Wnt/beta-catenin and P53 pathway activation," Gynecologic Oncology, vol. 126, no. 3, pp. 466-473, 2012.

[15] B. Weigelt, P. H. Warne, M. B. Lambros, J. S. Reis-Filho, and J. Downward, "PI3K pathway dependencies in endometrioid endometrial cancer cell lines," Clinical Cancer Research, vol. 19, no. 13, pp. 3533-3544, 2013.

[16] A. Pavlidou and N. F. Vlahos, "Molecular alterations of $\mathrm{PI} 3 \mathrm{~K} / \mathrm{Akt} / \mathrm{mTOR}$ pathway: a therapeutic target in endometrial cancer," The Scientific World Journal, vol. 2014, Article ID 709736, 9 pages, 2014.

[17] D. A. Iglesias, M. S. Yates, D. van der Hoeven et al., "Another surprise from metformin: novel mechanism of action via k-Ras influences endometrial cancer response to therapy," Molecular Cancer Therapeutics, vol. 12, no. 12, pp. 2847-2856, 2013.

[18] Y. Achiwa, K. Hasegawa, and Y. Udagawa, "Regulation of the phosphatidylinositol 3-Kinase-Akt and the mitogen-activated protein kinase pathways by ursolic acid in human endometrial cancer cells," Bioscience, Biotechnology and Biochemistry, vol. 71, no. 1, pp. 31-37, 2007.

[19] J. Pallarés, A. Velasco, N. Eritja et al., "Promoter hypermethylation and reduced expression of RASSF1A are frequent molecular alterations of endometrial carcinoma," Modern Pathology, vol. 21, no. 6, pp. 691-699, 2008.

[20] E. Castellano and J. Downward, "Ras interaction with PI3K: more than just another effector pathway," Genes \& Cancer, vol. 2, no. 3, pp. 261-274, 2011.

[21] E. Zwick, J. Bange, and A. Ullrich, "Receptor tyrosine kinase signalling as a target for cancer intervention strategies," EndocrineRelated Cancer, vol. 8, no. 3, pp. 161-173, 2001.

[22] S. Gatius, A. Velasco, A. Azueta et al., "FGFR2 alterations in endometrial carcinoma," Modern Pathology, vol. 24, no. 11, pp. 1500-1510, 2011.

[23] M. Katoh and H. Nakagama, "FGF receptors: cancer biology and therapeutics," Medicinal Research Reviews, vol. 34, no. 2, pp. 280-300, 2014.

[24] T. C. Harding, L. Long, S. Palencia et al., "Blockade of nonhormonal fibroblast growth factors by FP-1039 inhibits growth of multiple types of cancer," Science Translational Medicine, vol. 5, no. 178, Article ID 178ra39, 2013.

[25] G. E. Konecny, T. Kolarova, N. A. O’Brien et al., "Activity of the fibroblast growth factor receptor inhibitors dovitinib (TKI258) and NVP-BGJ398 in human endometrial cancer cells," Molecular Cancer Therapeutics, vol. 12, no. 5, pp. 632-642, 2013.

[26] T. C. Wehler, K. Frerichs, C. Graf et al., "PDGFR $\alpha / \beta$ expression correlates with the metastatic behavior of human colorectal cancer: a possible rationale for a molecular targeting strategy," Oncology Reports, vol. 19, no. 3, pp. 697-704, 2008.

[27] M. P. Pinto, W. W. D. Dye, B. M. Jacobsen, and K. B. Horwitz, "Malignant stroma increases luminal breast cancer cell proliferation and angiogenesis through platelet-derived growth factor signaling," BMC Cancer, vol. 14, no. 1, article 735, 2014.
[28] B. Liegl, C. Gülly, O. Reich, F. F. Nogales, A. Beham, and S. Regauer, "Expression of platelet-derived growth factor receptor in low-grade endometrial stromal sarcomas in the absence of activating mutations," Histopathology, vol. 50, no. 4, pp. 448452, 2007.

[29] F. Rosa, D. Hwang Corazon, J. Jerald et al., "Inhibition of platelet-derived growth factor receptor phosphorylation by STI571 (Gleevec) reduces growth and metastasis of human pancreatic carcinoma in an orthotopic nude mouse model," Clinical Cancer Research, vol. 9, no. 17, pp. 6534-6544, 2003.

[30] A. Östman and C.-H. Heldin, "PDGF receptors as targets in tumor treatment," Advances in Cancer Research, vol. 97, pp. 247274, 2007.

[31] A. Ferrajoli, S. Faderl, F. Ravandi, and Z. Estrov, "The JAK-STAT pathway: a therapeutic target in hematological malignancies," Current Cancer Drug Targets, vol. 6, no. 8, pp. 671-679, 2006.

[32] J.-P. Spano, G. Milano, C. Rixe, and R. Fagard, "JAK/STAT signalling pathway in colorectal cancer: a new biological target with therapeutic implications," European Journal of Cancer, vol. 42, no. 16, pp. 2668-2670, 2006.

[33] S. Y. Lai and F. M. Johnson, "Defining the role of the JAKSTAT pathway in head and neck and thoracic malignancies: implications for future therapeutic approaches," Drug Resistance Updates, vol. 13, no. 3, pp. 67-78, 2010.

[34] F. P. S. Santos and S. Verstovsek, "JAK2 inhibitors: what's the true therapeutic potential?" Blood Reviews, vol. 25, no. 2, pp. 53-63, 2011.

[35] L. Vijayakrishnan, R. Venkataramanan, and P. Gulati, “Treating inflammation with the Janus Kinase inhibitor CP-690,550," Trends in Pharmacological Sciences, vol. 32, no. 1, pp. 25-34, 2011.

[36] S. N. Constantinescu, M. Girardot, and C. Pecquet, "Mining for JAK-STAT mutations in cancer," Trends in Biochemical Sciences, vol. 33, no. 3, pp. 122-131, 2008.

[37] J. Gao, J. Tian, Y. Lv et al., "Leptin induces functional activation of cyclooxygenase-2 through JAK2/STAT3, MAPK/ERK, and PI3K/AKT pathways in human endometrial cancer cells," Cancer Science, vol. 100, no. 3, pp. 389-395, 2009.

[38] Y. Liu, L. Lv, W. Xiao et al., "Leptin activates STAT3 and ERK1/2 pathways and induces endometrial cancer cell proliferation," Journal of Huazhong University of Science and Technology Medical Sciences, vol. 31, pp. 365-370, 2011. 


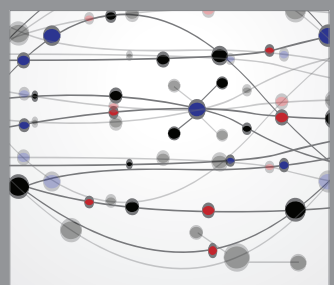

The Scientific World Journal
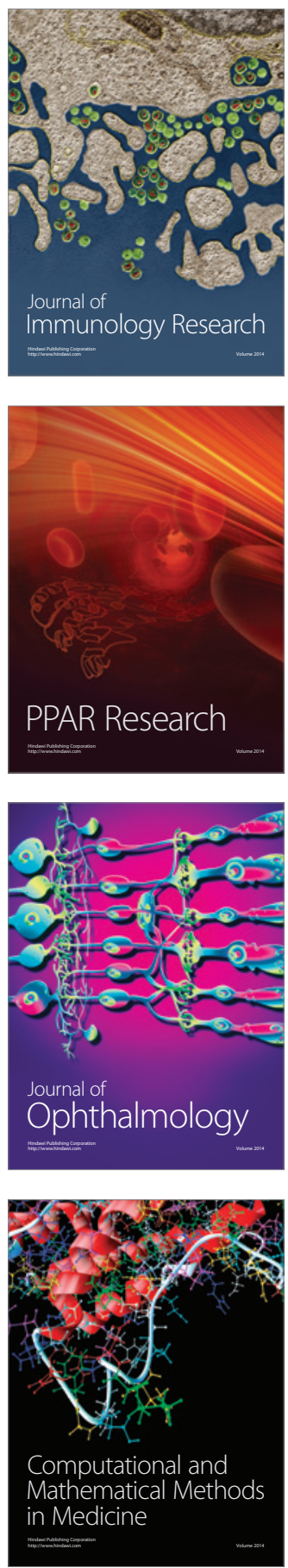

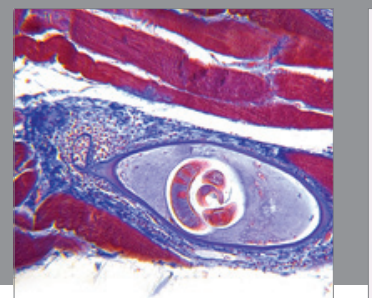

Gastroenterology

Research and Practice
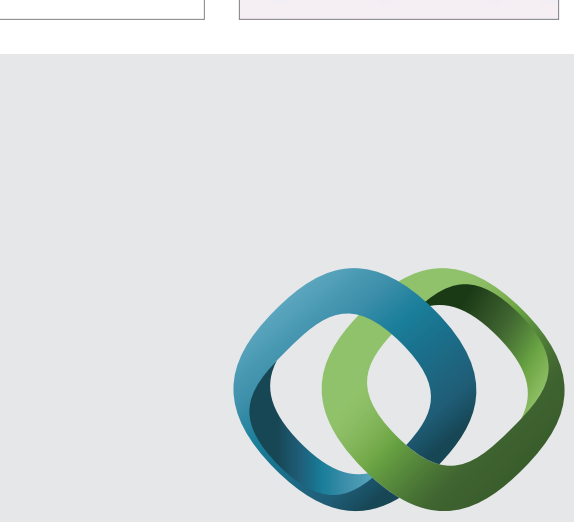

\section{Hindawi}

Submit your manuscripts at

http://www.hindawi.com
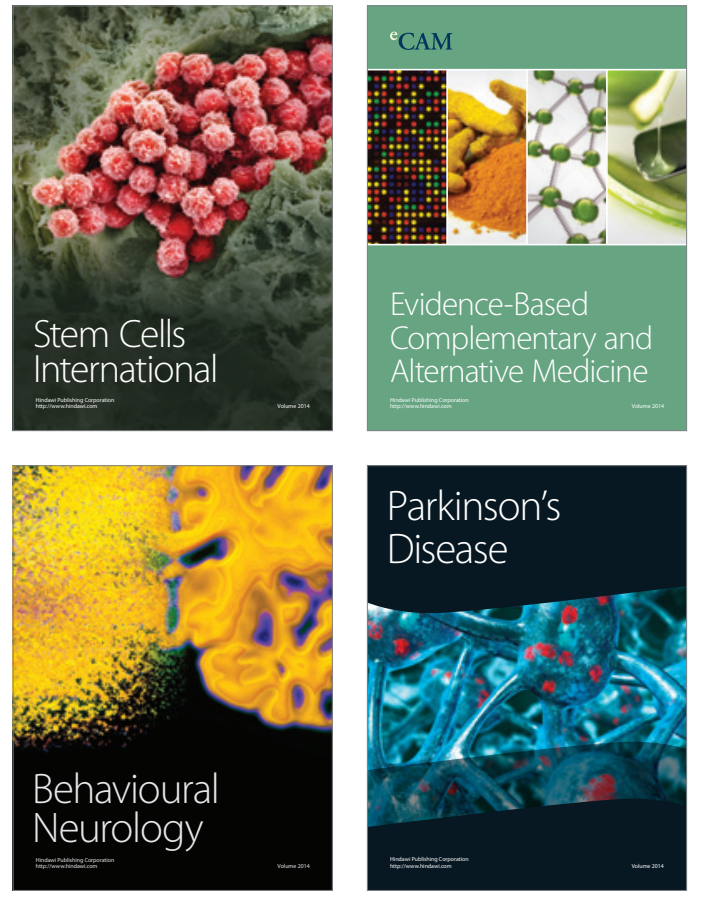
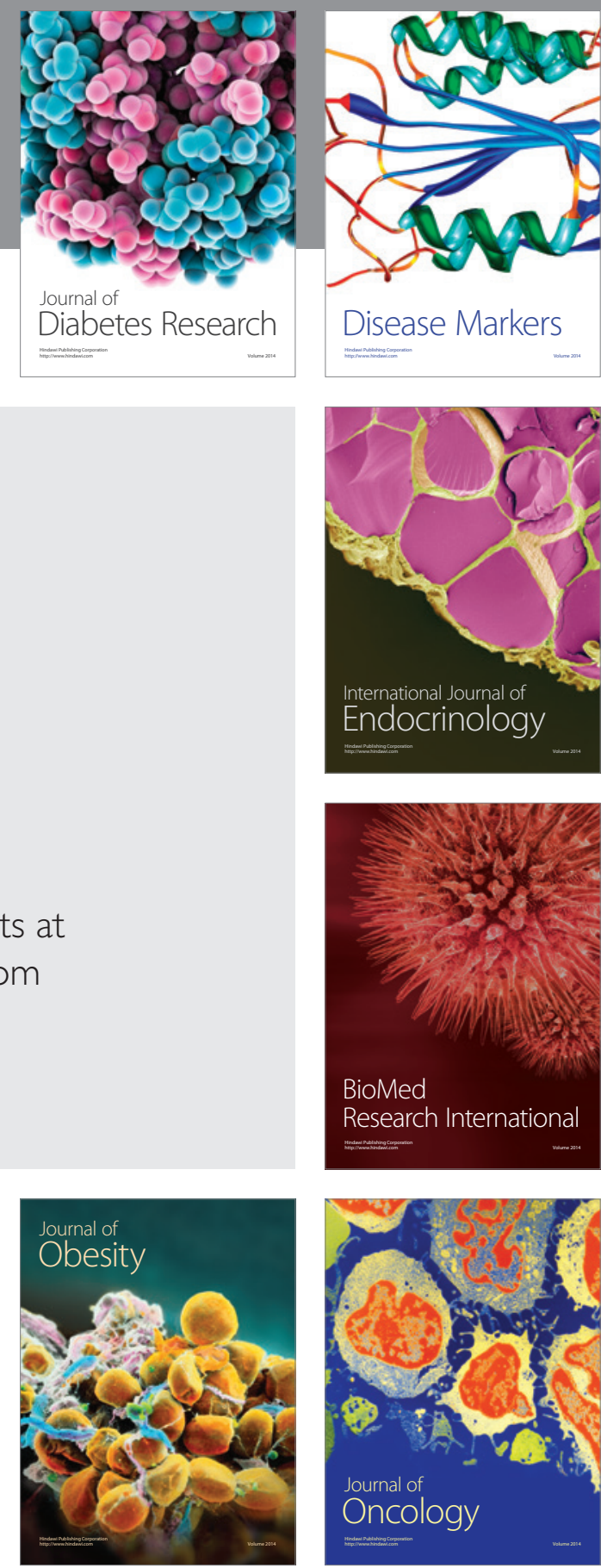

Disease Markers
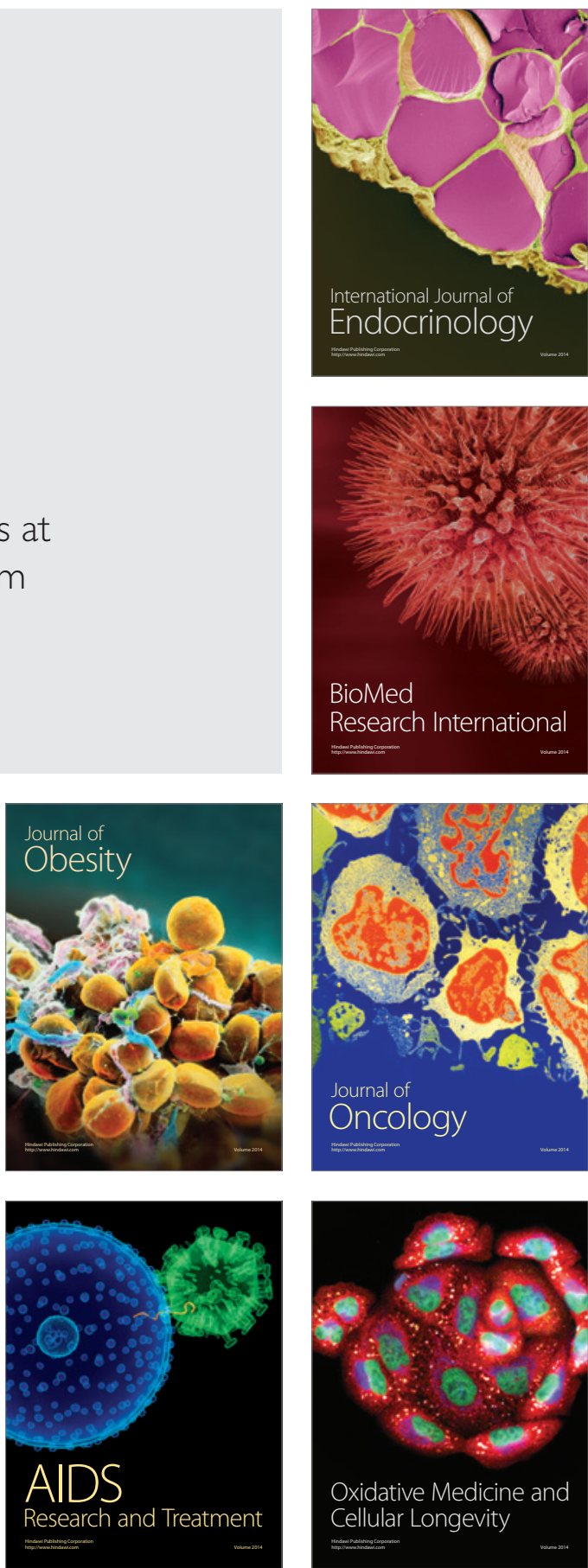